\title{
Clostridium tertium
}

National Cancer Institute

\section{Source}

National Cancer Institute. Clostridium tertium. NCI Thesaurus. Code C86297.

A species of anaerobic, Gram positive, rod shaped bacteria assigned to the phylum Firmicutes. This species is aerotolerant, non-exotoxin producing, ferments glucose, lactose, maltose and sucrose, reduces nitrate and is nonproteolytic. C. tertium has traditionally been considered nonpathogenic but has since been designated an emerging pathogen in immunocompromised and wound patients, causing necrotizing fasciitis and gas gangrene. 